\title{
High-throughput cis-regulatory element discovery in the vector mosquito Aedes aegypti
}

\author{
Susanta K. Behura, ${ }^{1,2 \dagger}$, Joseph Sarro ${ }^{1,2,3+}$, Ping Li $i^{1,3}$, Keshava Mysore ${ }^{1,3}$, David W. Severson ${ }^{1,2,3}$,
} Scott J. Emrich ${ }^{1,2,4^{*}+}$ and Molly Duman-Scheel ${ }^{1,2,3^{*}+}$

\begin{abstract}
Background: Despite substantial progress in mosquito genomic and genetic research, few cis-regulatory elements (CREs), DNA sequences that control gene expression, have been identified in mosquitoes or other non-model insects. Formaldehyde-assisted isolation of regulatory elements paired with DNA sequencing, FAIRE-seq, is emerging as a powerful new high-throughput tool for global CRE discovery. FAIRE results in the preferential recovery of open chromatin DNA fragments that are not bound by nucleosomes, an evolutionarily conserved indicator of regulatory activity, which are then sequenced. Despite the power of the approach, FAIRE-seq has not yet been applied to the study of non-model insects. In this investigation, we utilized FAIRE-seq to profile open chromatin and identify likely regulatory elements throughout the genome of the human disease vector mosquito Aedes aegypti. We then assessed genetic variation in the regulatory elements of dengue virus susceptible (Moyo-S) and refractory (Moyo-R) mosquito strains.
\end{abstract}

Results: Analysis of sequence data obtained through next generation sequencing of FAIRE DNA isolated from A. aegypti embryos revealed $>121,000$ FAIRE peaks (FPs), many of which clustered in the $1 \mathrm{~kb} 5^{\prime}$ upstream flanking regions of genes known to be expressed at this stage. As expected, known transcription factor consensus binding sites were enriched in the FPs, and of these FoxA1, Hunchback, Gfi, Klf4, MYB/ph3 and Sox9 are most predominant. All of the elements tested in vivo were confirmed to drive gene expression in transgenic Drosophila reporter assays. Of the $>13,000$ single nucleotide polymorphisms (SNPs) recently identified in dengue virus-susceptible and refractory mosquito strains, 3365 were found to map to FPs.

Conclusion: FAIRE-seq analysis of open chromatin in A. aegypti permitted genome-wide discovery of CREs. The results of this investigation indicate that FAIRE-seq is a powerful tool for identification of regulatory DNA in the genomes of non-model organisms, including human disease vector mosquitoes.

Keywords: Genome, FAIRE-seq, Dengue virus, Zika, Next generation sequencing, Drosophila

\section{Background}

Vector mosquitoes inflict more human suffering than any other organism and spread diseases that kill more than one million people each year. Mosquito-borne illnesses, among the most complex infectious diseases to

\footnotetext{
* Correspondence: semrich@nd.edu; mscheel@nd.edu

${ }^{\dagger}$ Equal contributors

${ }^{1}$ Eck Institute for Global Health, University of Notre Dame, Notre Dame, IN 46556, USA

Full list of author information is available at the end of the article
}

prevent and control, have resurged worldwide and pose threats for epidemic outbreaks in developed countries, including the United States. Given poor progress in vaccine development and distribution, mosquito control is the primary mechanism for disease control. However, the emergence of insecticide resistance and a lack of support for mosquito control programs compromise strategies for managing mosquitoes, resulting in a need for the development of new approaches to combat these insect vectors of human disease [1]. The mosquito genome projects have facilitated research in 
many new facets of mosquito biology [2-5]. However, despite substantial progress in mosquito genomic and genetic research, very few cis-regulatory elements (CREs), DNA sequences that control gene expression, have been identified in the mosquito genomes. CRE discovery is challenging because these elements are typically short sequences contained within vast stretches of intergenic DNA [6]. Although computational approaches resulted in the identification of a number of mosquito CREs [6], CRE discovery has not continued to progress, and regulatory regions for most mosquito genes remain unknown. This deficiency-a significant gap in our basic knowledge of mosquito genetics-has resulted in a lack of drivers to manipulate gene expression in selected tissues at specific times, the inability to properly dissect gene regulatory networks in mosquitoes, and difficulty in understanding the biological meaning of genetic variation that resides in non-coding regions.

FAIRE-seq, formaldehyde-assisted isolation of regulatory elements paired with DNA sequencing [7], is emerging as a powerful new approach for global biochemical isolation of CREs through their lack of association with nucleosome proteins. FAIRE, which exploits the ability of formaldehyde exposure to form crosslinks between interacting nucleosomal histones and DNA, has emerged as a powerful new approach for genome-wide identification of regulatory elements [7-9]. During the FAIRE process, chromatin is cross-linked with formaldehyde, sheared, and then phenol-chloroform extracted, permitting recovery of open chromatin DNA fragments that are not bound by nucleosomes, an evolutionarily conserved indicator of regulatory activity [10]. FAIRE has many advantages over alternative methods, one being that the recent pairing of this technique with nextgeneration sequencing, FAIRE-seq, permits straightforward and genome-wide high throughput detection of CREs [7]. FAIRE-seq is technically straightforward and involves fewer steps, reagents, and variables than alternative methodologies. Use of FAIRE eliminates the tedious titration of DNAse activity and limits concerns about variations in nuclei preparations that affect other protocols. Unlike chromatin immunoprecipitation, there is no dependence on antibody supply/quality or the production of tagged proteins. Also, unlike DNAse sensitivity assays, FAIRE requires no cellular treatments prior to crosslinking and better captures the endogenous chromatin state [8].

Here, we describe a FAIRE-seq investigation in the human disease vector mosquito Aedes aegypti. A. aegypti, a container-breeding mosquito that is closely associated with humans and their urban dwellings, transmits viruses that cause Zika, yellow fever, chikungunya, and dengue, the most widespread and significant arboviral disease in the world. A third of the world's population is at risk for dengue virus (DENV) infection, a leading cause of illness and death in the tropics and subtropics. DENV is transmitted to as many as 400 million people each year when they are bitten by infected Aedes mosquitoes [1]. A. aegypti strains that are susceptible (Moyo-S) and refractory (Moyo-R) to DENV infection have been selected from a common genetic background [11, 12]. Genome-wide transcriptome comparisons suggest that modular expression of genes is significantly different in the two strains and that many genes ( 2500) may contribute to susceptible/refractory responses of $A$. aegypti to DENV infection [13]. In a companion study, next generation sequencing identified $>13,000$ single nucleotide polymorphisms (SNPs) in the two strains [14-16]. In this investigation, FAIRE-seq analysis of open chromatin in A. aegypti permitted genome-wide discovery of cis-regulatory elements, which facilitated analysis of genetic variation in noncoding cis-regulatory elements that may contribute to mosquito susceptibility and responses to DENV infection.

\section{Methods}

Ethics

This study did not include human subjects, human data, or vertebrate animals.

\section{Mosquito rearing and egg collection}

The A. aegypti Liverpool-IB12 (LVP-IB12) strain, from which the genome sequence [3] was derived, was used in this investigation. Mosquitoes were maintained in an insectary at $26{ }^{\circ} \mathrm{C}, \sim 80 \%$ humidity, under a $12 \mathrm{~h}$ light and $12 \mathrm{~h}$ dark cycle with $1 \mathrm{~h}$ crepuscular periods at the beginning and end of each light cycle. Larvae fed on a suspension of dried beef liver powder, and adults were provided cotton soaked with $10 \%$ sugar solution. For blood feeding adult females, an artificial membrane feeding system was used in conjunction with sheep blood purchased from Hemo-Stat Laboratories (Dixon, CA). Females deposited eggs on paper toweling during twohour eggs collections. Eggs were maintained in the insectary for an additional $49 \mathrm{~h}$.

\section{FAIRE}

DNA was prepared using a modified version of the Drosophila melanogaster embryonic tissue protocol [17], which is based on Simon et al. [7] methodology. DNA processed in this manner will hereafter be referred to as FAIRE DNA. In summary, $100 \mathrm{mg}$ of eggs were treated with $50 \%$ bleach for $3 \mathrm{~min}$. and then rinsed thoroughly with distilled water. Crosslinking was performed for 15 min using $10 \mathrm{~mL} 0.4 \%$ formaldehyde in PEM buffer (100 mM PIPES disodium salt, $2 \mathrm{mM}$ EGTA, $1 \mathrm{mM}$ magnesium sulfate; $\mathrm{pH} 7$ ) in a $60{ }^{\circ} \mathrm{C}$ water bath. Following crosslinking, which was quenched through addition 
of glycine, embryonic nuclei were pelleted through centrifugation at $1500 \mathrm{RCF}$ at $4{ }^{\circ} \mathrm{C}$ for $2 \mathrm{~min}$. The nuclei were resuspended in Buffer A (10 mM HEPES pH 8, 10 mM EDTA, 0.5 mM EGTA, $0.25 \%$ Triton X-100) and homogenized with a pestle. The lysate was passed through Miracloth to remove debris. The nuclei were pelleted again, resuspended in FAIRE lysis buffer ( $2 \%$ Triton X-100, 1 \% SDS, $100 \mathrm{mM} \mathrm{NaCl}, 10 \mathrm{mM}$ Tris-Cl $\mathrm{pH} 8,1 \mathrm{mM}$ EDTA), and subjected to six rounds of bead beating with $0.5 \mathrm{~mm}$ glass beads/vortexing at $4{ }^{\circ} \mathrm{C}$. Chromatin was sonicated using a Branson 250 ultrasonifier outfitted with a microtip $(6 \times 30 \mathrm{~s}$ with $1 \mathrm{~s}$ pulse, $0.5 \mathrm{~s}$ stop, $22 \%$ amplitude), generating a size range of 300-500 bp fragments. Soluble chromatin was then recovered following centrifugation at 15,000 G for $5 \mathrm{~min}$ at $4{ }^{\circ} \mathrm{C}$. FAIRE DNA was recovered from the cell lysate through phenol chloroform extraction in which openchromatin DNA was recovered from the aqueous phase. The recovered open chromatin was treated with RNAse A and proteinase $\mathrm{K}$, and then purified through a Qiaquick spin-column (Qiagen, Valencia, CA). The yield of FAIRE DNA $(\sim 1 \mu \mathrm{g})$ was sufficient for sequencing and corresponded to $\sim 1 \%$ of total DNA, well within the acceptable yield recommended by Simon et al. [7].

\section{Illumina library construction and sequencing}

FAIRE DNA Illumina libraries were prepared by the University of Notre Dame Genomics and Bioinformatics Core facility using the TruSeq kit (Illumina, San Diego, CA) per the manufacturer's guidelines. $1 \mu \mathrm{g}$ of FAIREenriched DNA was processed following the Illumina TruSeq DNA LT sample preparation protocol. Briefly, samples were end repaired and 3' adenylated. Illumina adapters were ligated to the template, then purified and size-selected (for 100-300 bp fragments) on an agarose gel. A 10-cycle PCR reaction enriched for ligation products containing both Illumina adapters. Sample concentration was measured on a Qubit fluorometer (Life Technologies, Grand Island, NY), and sample size distribution was assayed on an Agilent 2100 Bioanalyzer (Agilent Technologies, Inc., Santa Clara, CA). Next-generation Illumina Sequencing (HiSeq 50 bp paired-end sequencing) with an Illumina HiSeq 2000 (BGI Americas Corporation, Cambridge, MA) was used to generate $\sim 150$ million reads for each of three replicate FAIRE DNA preparations.

\section{Analysis of FAIRE sequencing data}

Raw sequences were trimmed of adapters with Trimmomatic [18] version 0.32 and assessed for quality with FastQC [19] version v0.10.1. Trimmed sequences were aligned to version three of the $A$. aegypti scaffolds reference genome $[20,21]$ using the backtrack algorithm in BWA [22] version 0.5.9-r16 for three replicates. The third replicate, however, required further processing due to an unknown segmentation fault. A subset of fifty thousand reads containing one or more reads that could not be processed by BWA was located using Makeflow_BWA [23] and removed to enable successful alignment. Reads were further filtered with Samtools [22] before checking for reproducibility of replicates with the Irreproducible Discovery Rate (IDR) framework as described [24, 25] and calling peaks with MACS2 [26]. These peaks are hereafter referred to as FAIRE peaks (FPs). The maximum insert size itself was estimated empirically using BWA and resulted in calling peaks with an extent size of $550 \mathrm{bp}$.

\section{Prediction of known transcription factor binding sites}

Overrepresentation analysis of FPs for known transcription factor binding sites (JASPAR eukaryote, data downloaded in March 2014) was performed. The 'Clover' (Cis-eLement OVERrepresentation) tool [27] was used to determine motif incidences in the FPs. In the first step, the degree of motif [transcription factor (TF)-binding] incidences in the FPs was expressed by a score that was calculated. In the second step, the $p$-value was obtained from the raw score, which is essentially the probability that the motif incidence score is either equal or greater to that of the threshold (user defined, set to a default value of 6) by chance. The background sequences were generated for Clover analysis as described previously [28].

\section{Association of FPs with gene expression data in embryos}

To determine if FP sequences identified in this study are associated with immediate $(<1 \mathrm{~kb}) 5^{\prime}$ upstream regulatory regions of genes expressed during this embryonic stage, FPs were compared with available $48-52 \mathrm{~h}$ embryonic transcriptome data previously described by Akbari et al. [29].

\section{Identification of SNPs in FPs}

Single nucleotide polymorphisms (SNPs) within FP sequences were determined from a set of SNP data generated from a companion study (which will be described in its entirety elsewhere) in which Illumina sequencing of genomic DNA from two A. aegypti lab strains, Moyo-S and Moyo-R, was performed [14-16]. Both strains have been described previously $[11,12]$. The SNP positions predicted within each supercontig were used to assess if they are present within the FP start and end coordinates within the same supercontigs.

\section{Analysis of genomic distribution of FPs}

The map locations of FPs were compared relative to the genomic coordinates of gene models (both protein coding and non-coding genes) to determine their associations. All 3' and 5' untranslated region (UTR) sequences of $A$. aegypti genes were obtained from VectorBase (VB) 
[20, 21], and the UTR regulatory elements present in those sequences were identified by using UTRScan [30].

\section{Transgenic reporter generation and analysis}

FP DNA sequences of interest were PCR-amplified from A. aegypti genomic DNA and cloned into plasmid pattBnucGFPs, a $\phi C 31$-enabled Drosophila transformation vector containing enhanced green fluorescent protein (EGFP) under the control of a minimal $h s p 70$ promoter (graciously provided by M.S. Halfon). Transgenic Drosophila were produced at Rainbow Transgenic Flies, Inc. (Camarillo, CA) by injection into line PBac\{y[+]-attP-9A\}VK00027 (Bloomington stock 9744 [31]). Tissue from transgenic animals was collected and fixed as described previously [32]. Tissues were mounted and imaged on a Zeiss 710 confocal microscope using Zen software or a Zeiss Axioimager equipped with a Spot Flex camera and Spot Digital Imaging software. Images were processed with Adobe Photoshop software.

\section{Results and discussion}

\section{Gene-centric distribution of FPs}

FAIRE-seq open chromatin profiling was performed to identify regulatory DNA in the $A$. aegypti genome. For these studies, embryos were collected $50+/-1 \mathrm{~h}$ after eggs were laid, a time point that coincides with the onset of axon pathfinding [33], a topic of interest to our laboratory [33-35], and a time period for which transcriptome data are available [29]. The FAIRE procedure was optimized using these $A$. aegypti embryos as input tissue until a $1 \%$ FAIRE DNA yield (with respect to the total input DNA) was obtained, an amount that is within the optimal range noted by Simon et al. [7]. FAIRE-seq replicate experiments were highly reproducible, as evidenced by IDR [24, 25] analysis (Additional file 1) which detected no significant differences between three replicate experiments, the data from which were subsequently combined for downstream analyses. FAIRE-seq reads [36] were mapped to version three of the $A$. aegypti scaffolds reference v.4 $[20,21]$ and have been made accessible through the VB Genome Browser (Fig. 1). In total, FAIRE-seq open chromatin profiling resulted in the identification of $\sim 121,600$ FPs (Additional file 2) [36], a number which is consistent with results reported in other systems [7].

\section{FAIRE-seq identifies regulatory elements in the $A$. aegypti genome}

Several lines of evidence indicate that FAIRE reliably identified regulatory elements in A. aegypti. In total, $5 \%$ of the FPs were found to be located at proximal promoters (Additional file 3), a figure that is consistent with reports in other systems $[37,38]$. These sequences can include both core promoters as well as regulatory

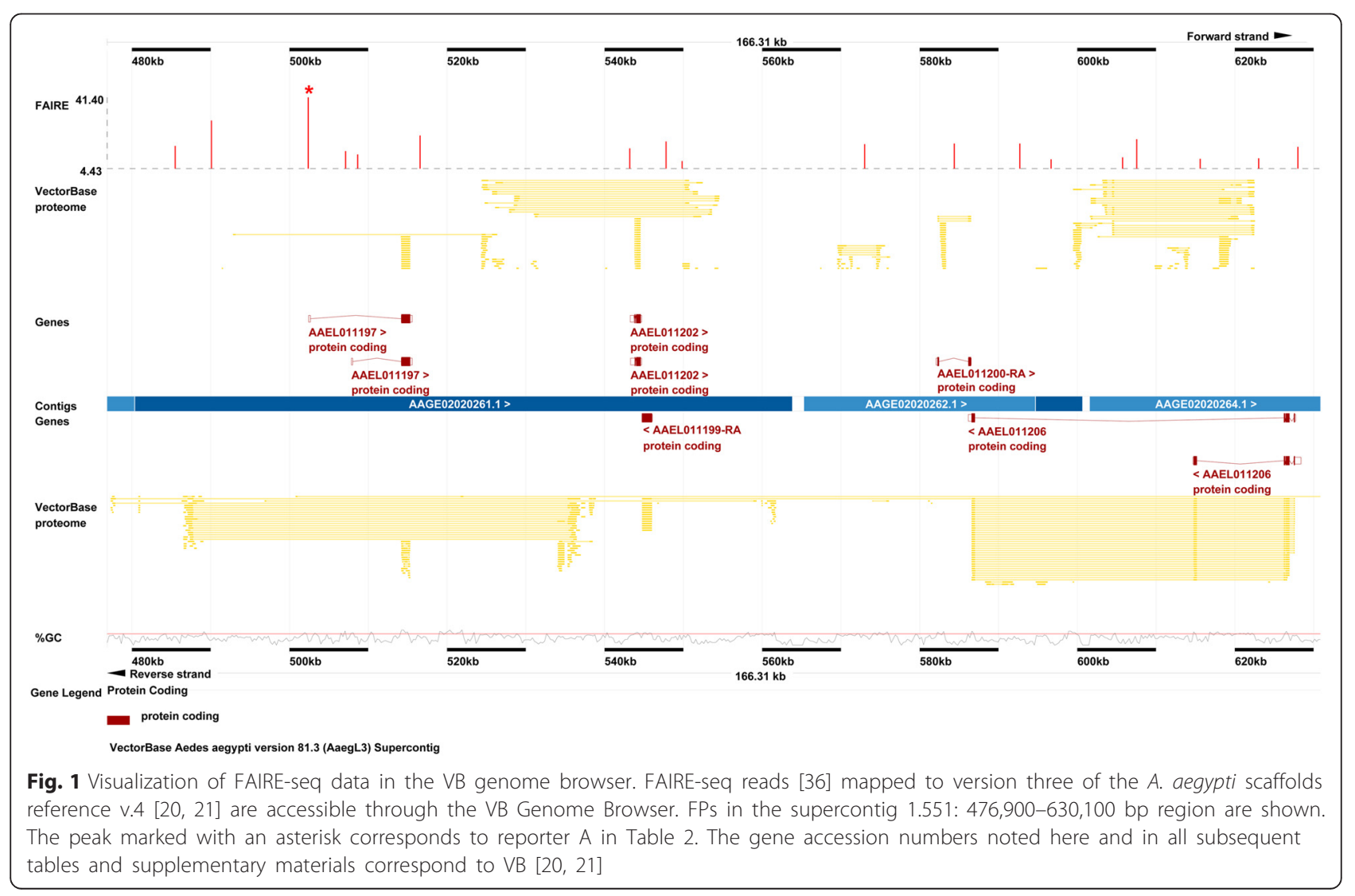


elements adjacent to the promoters. However, one limitation of FAIRE is that other assays, including DNAse-seq, may be better for identification of nucleosome-depleted promoters of highly expressed genes [7, 38]. The aggregated FAIRE signal around all transcription start sites (TSSs) showed higher frequency within 100-200 bp upstream of TSSs (Fig. 2), which is also expected [7]. Furthermore, transcription factor binding sites are often associated with regulatory sequences in eukaryotes [39]. Known transcription factor binding sites are enriched in the FPs (Table 1) with respect to the rest of the genome. Among them, the top 20 TFbinding sites that are significantly enriched in FPs are shown in Fig. 3. Of these, the following consensus sequences are most abundant: FoxA1, Gfi, Hunchback, Klf4, $\mathrm{MYB} / \mathrm{ph} 3$ and Sox9. Some of these elements, particularly Hunchback and FoxA1, were also abundant in FPs localized to the UTRs and intragenic regions (these regions are discussed further below).

Next, the proximity of FPs with respect to genes known to be expressed in $A$. aegypti embryos was examined. The developmental transcriptome of $A$. aegypti was recently described [29]. RNA-seq data is available for 48-52 h embryos [29], which includes the time span analyzed in the FAIRE-seq study (49-51 h). Analysis of the FAIRE data with respect to RNA-seq data revealed that $78 \%$ (4733) of genes expressed at this developmental stage have a 5 ' flanking (within $1 \mathrm{~kb}$ upstream of the TSS) or intragenic FP. In the majority of cases (89\%), these FPs are located within $1 \mathrm{~kb}$ upstream of the TSS of the gene, and in the other $11 \%$ of cases, they reside within the genes in question. For cases in which FPs localized to within 100 bp upstream of the TSS, the gene has a relatively higher expression level (1.4 fold) compared to genes for which FPs were localized to more distant locations. This suggests that positional proximity of regulatory sequences may influence expression of the

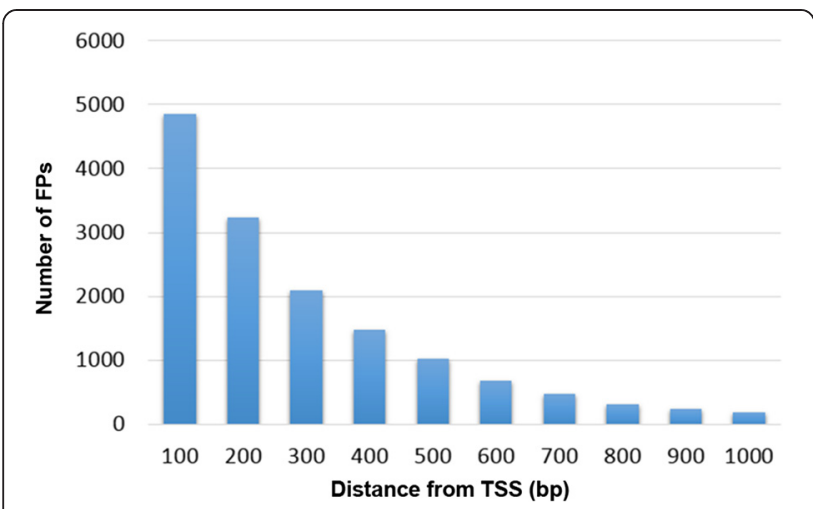

Fig. 2 FPs are enriched around TSSs. The aggregated FAIRE signal adjacent to transcription start sites (TSSs) was increased within 100-200 bp upstream of TSSs
Table 1 Consensus binding sites enriched in FPs

\begin{tabular}{|c|c|}
\hline TF binding & Motif incidences \\
\hline CG11617 & 2813 \\
\hline FOXA1 & 1225 \\
\hline SRY & 1222 \\
\hline Optix & 901 \\
\hline $\mathrm{H} 2.0$ & 845 \\
\hline Prrx & 813 \\
\hline Ubx & 773 \\
\hline ARID3A & 723 \\
\hline Onecut & 678 \\
\hline $\mathrm{ct}$ & 670 \\
\hline Hunchback & 641 \\
\hline cad & 538 \\
\hline CG42234 & 538 \\
\hline PHDP & 481 \\
\hline NFIC & 345 \\
\hline Lim3 & 338 \\
\hline hbn & 330 \\
\hline bap & 312 \\
\hline slp1 & 312 \\
\hline Athb-1 & 222 \\
\hline SPI1 & 210 \\
\hline CG4328 & 204 \\
\hline E5 & 194 \\
\hline Lhx3 & 165 \\
\hline CG34031 & 160 \\
\hline Dr & 160 \\
\hline DIl & 155 \\
\hline HIF1A:ARNT & 135 \\
\hline CF2-II & 133 \\
\hline Vsx2 & 109 \\
\hline zen2 & 105 \\
\hline
\end{tabular}

Known transcription factor binding sites were found to be enriched in FPs (with respect to the entire A. aegypti genome). Motif incidences in FPs (>100) as determined by Clover are shown here. In each case, Clover shows a significant $p$ value for enrichment of the motif in FPs with the genome sequence used as background sequences for comparison

downstream genes, at least during embryonic development in $A$. aegypti.

To further assess whether FAIRE-seq identified regulatory regions in the $A$. aegypti genome, a number of FP DNA sequences were tested for their ability to drive gene expression in vivo. Although transgenic technology is available in $A$. aegypti, transgenic generation in $D$. melanogaster is mature, quick, and inexpensive, and it is relatively easier and cheaper to maintain fruit fly stocks. Given the merits of Drosophila transgenics, $11 \mathrm{~A}$. aegypti FP DNA sequences (Table 2) were cloned into a 


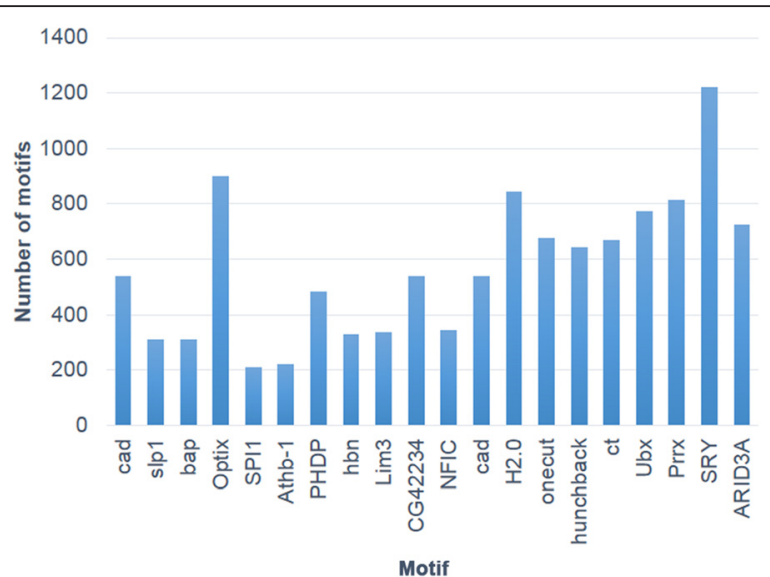

Fig. 3 Top TF-binding sites significantly enriched in FPs. The top 20 TF-binding sites that are significantly enriched in the FPs are shown

Drosophila transformation vector containing EGFP under the control of a minimal $h s p 70$ promoter. $100 \%$ of these elements were confirmed to drive EGFP reporter expression in D. melanogaster (Fig. 4). Although the FAIRE-seq study was performed in A. aegypti embryos, in addition to embryos (Fig. 4a, b), a number of the FP sequences identified were found to be capable of driving gene expression at later stages of the Drosophila life cycle, including the larval (Fig. 4c, e, f, i, j, k), pupal (Fig. 4d), and adult (Fig. 4g, h) stages. These findings suggest that some of the regulatory sequences that function in embryos are also active at later stages of the life cycle, including adults. However, a recent FAIRE-seq study in D. melanogaster showed that following celltype specification in the appendages, open chromatin profiles changed as the appendages progressed toward terminal differentiation, suggesting that stage-specific functions also require opening of new regulatory sites or the closing of existing sites [17]. In the future, it will be interesting to generate $A$. aegypti transgenic reporters to examine the activity of the regulatory elements assessed here in Drosophila (Table 2, Fig. 4) at multiple stages of the mosquito life cycle.

This investigation identified regulatory elements that drive gene expression in a wide variety of $D$. melanogaster tissues, including the eye (Fig. 4d), gut (Fig. 4b, e), salivary gland (Fig. 4c), fat body (Fig. 4f), olfactory system (Fig. 4g, h), denticle belts (Fig. 4i), leg imaginal discs (Fig. 4j), and brain (Fig. 4k). These studies in transgenic fruit flies provide functional validation of the FAIRE-seq data, suggesting that this investigation has succeeded in the goal of identifying regulatory elements. The Drosophila reporter assays permitted high throughput analysis of $A$. aegypti FAIRE-seq data in a manner which is not presently possible in mosquitoes, but which will lay the groundwork for full characterization of select CREs of interest directly in A. aegypti in future studies. To this end, we are presently pursuing a screen in Drosophila that aims to identify FP sequences that promote gene expression in tissues of vector importance (i.e., the olfactory system, midgut, salivary gland, and fat bodies). The elements selected for transgenic reporter studies presented here (Table 2, Fig. 4) were chosen based on their potential for driving gene expression in these tissues, which was estimated largely through examination of available Drosophila or mosquito gene expression data. Following the Drosophila screen, a number of regulatory elements that are confirmed to promote gene expression in tissues of interest will be tested directly in A. aegypti transgenics. It will be interesting to determine if these drivers promote comparable gene expression patterns in both $D$. melanogaster and A. aegypti. It is anticipated that if $A$. aegypti CREs drive tissue-specific reporter expression in Drosophila that mimics the activity of these

Table 2 FAIRE transgenic reporters

\begin{tabular}{|c|c|c|c|c|}
\hline Reporter & $\mathrm{FP}$ & Enrichment $p$-value & Flanks VB Gene \# & Gene TSS \\
\hline $\mathrm{A}$ & Supercont1.551:501192-503018 & $8.27104 \mathrm{E}-39$ & AAEL011197 & Supercont1.551: 507860 \\
\hline $\mathrm{B}$ & Supercont1.440:550819-551917 & 2.40519E-06 & AAEL009947 & Supercont1.440: 551773 \\
\hline C & Supercont1.381:720103-720682 & 0.001345953 & AAEL009224 & Supercont1.381: 720570 \\
\hline $\mathrm{D}$ & Supercont1.2641:1068-1902 & 1.61139E-10 & AAEL015489 & Supercont1.2641: 1778 \\
\hline $\mathrm{E}$ & Supercont 1.911:297903-298590 & 3.79289E-08 & AAEL013757 & Supercont1.911: 294736 \\
\hline $\mathrm{F}$ & Supercont1.16:273854-274851 & $7.14776 \mathrm{E}-11$ & AAEL000765 & Supercont1.16: 283224 \\
\hline G & Supercont1.237:1279560-1280173 & 0.000437431 & AAEL007110 & Supercont1.237: 1269860 \\
\hline $\mathrm{H}$ & Supercont1.174:341062-341799 & $3.97961 \mathrm{E}-10$ & AAEL005776 & Supercont1.174: 357279 \\
\hline । & Supercont1.128:2089446-2090042 & 0.000177296 & AAEL004719 & Supercont1.128: 2098660 \\
\hline J & Supercont 1.635:654750-655775 & $6.27856 \mathrm{E}-26$ & AAEL011943 & Supercont1.635: 655775 \\
\hline K & Supercont1.160:604315-605761 & 1.43933E-45 & AAEL005507 & Supercont1.160: 435622 \\
\hline
\end{tabular}

The FP DNA sequences listed were tested for their ability to drive GFP reporter expression in D. melanogaster transgenics. These reporter assays confirmed that all of the indicated sequences function as regulatory elements (see Fig. 4 for results). The $p$-values for enrichment, flanking genes, and TSSs of the flanking genes are noted 


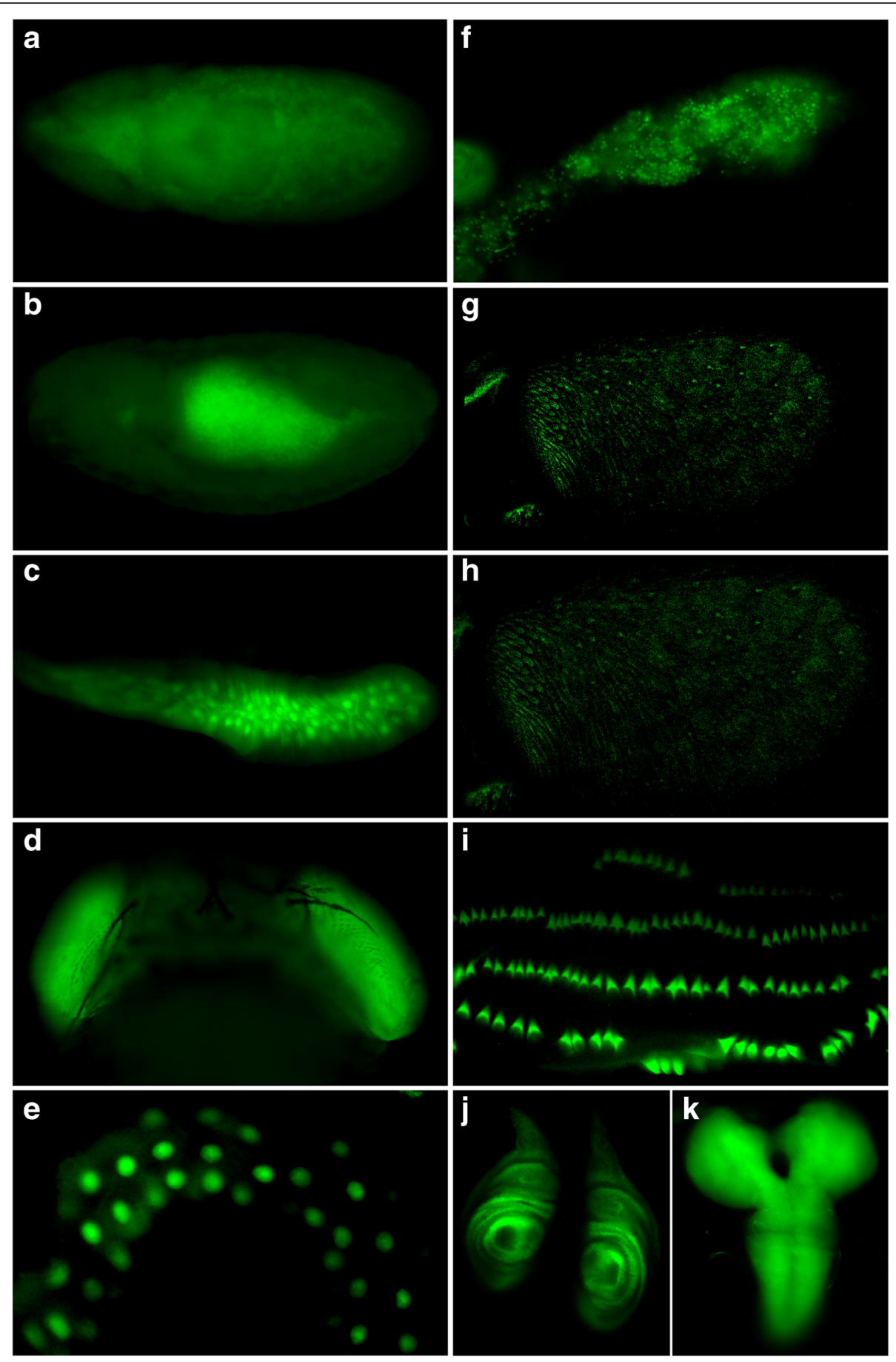

Fig. 4 FP DNA sequences promote gene expression in vivo. GFP reporter expression (from the reporters indicated in Table 2) was detected in the following D. melanogaster tissues: embryo (reporter A in $\mathbf{a}$; whole-mount is shown), embryonic midgut (reporter $B$ in $\mathbf{b}$ ), third instar larval salivary gland (reporter $\mathbf{C}$ in $\mathbf{c}$ ), pupal eyes (reporter $\mathrm{D}$ in $\mathbf{d}$ ), third instar larval gut (reporter $\mathbf{E}$ in $\mathbf{e}$ ), third instar larval fat body (reporter $\mathrm{F}$ in $\mathbf{f}$ ), adult antenna (reporter $\mathrm{G}$ in $\mathbf{g}$, reporter $\mathrm{H}$ in $\mathbf{~}$ ), third instar larval denticle belts (reporter I in i), third instar larval leg discs (reporter J in $\mathbf{j}$ ), and the third instar larval brain (reporter $\mathrm{K}$ in $\mathbf{k}$ )

elements in $A$. aegypti, the CREs are likely to function similarly in other dipterans, including additional vector mosquito species that are more closely related to $A$. aegypti than are fruit flies.
FPs mapping to UTRs

The 5' UTR, the mRNA leader sequence directly upstream of the translation initiation codon, plays critical roles in the regulation of transcription as well as 
translation in eukaryotes [40]. The regulatory roles of 3' UTRs are also well documented [41]. Using the UTRScan tool [30], a total of 405 and 764 FPs were identified in the 5'- (Additional file 4) and 3'- (Additional file 5) UTRs of genes, respectively. Interestingly, UTR-linked FPs appear to have a biased composition of known regulatory elements. Sequences representing the upstream open reading frame (uORF), Musashi-binding element (MBE), polyadenylation signal (PAS), and internal ribosome entry site (IRES) represented the most frequent regulators based on rank order analysis of their frequencies (Fig. 5). Interestingly, approximately half of all the UTR regulatory elements identified by UTRScan represented uORF elements. These elements, which are found in the $5^{\prime}$ region of a mRNA transcript, are capable of regulating protein production and impact organismal development and growth in fungi, plants, and animals, including insects [42].

\section{Intragenic regulators}

Although intragenic regulators are significant in mammalian genomes [43, 44], such regulatory elements remain poorly understood in arthropods. However, a recent genome-wide study in $D$. melanogaster has revealed that many enhancers are localized within genes [45]. To know if FPs in A.aegypti coincide with intragenic enhancers, FPs localized within genes (Additional file 6) were analyzed. 767 FPs were identified in the exons of 744 genes (Additional file 7), and a total of 4434 identified FPs were localized within the intron sequences of 3747 genes (Additional file 8). 3991 FPs were identified in the exonintron boundaries of 3592 genes (Additional file 9). The aggregated percentage of intragenic FPs is about $7.5 \%$ of all the FPs identified in the genome. The relative distribution of FPs in upstream vs. intragenic sites is similar to the distribution bias of enhancer elements in D. melanogaster genes [45], indicating possible evolutionary conservation of intragenic regulation of genes in dipterans.

\section{Association of FPs with non-coding genes}

Major classes of $A$. aegypti non-coding genes including tRNA, rRNA, snRNA, and microRNAs, also have FPs within $1 \mathrm{~kb}$ upstream of their TSSs (Fig. 6; Additional file 10). The precursor transcripts of miRNA genes are known to be regulated by cis-acting elements [46, 47], thus suggesting that FPs identified within $1 \mathrm{~kb}$ upstream

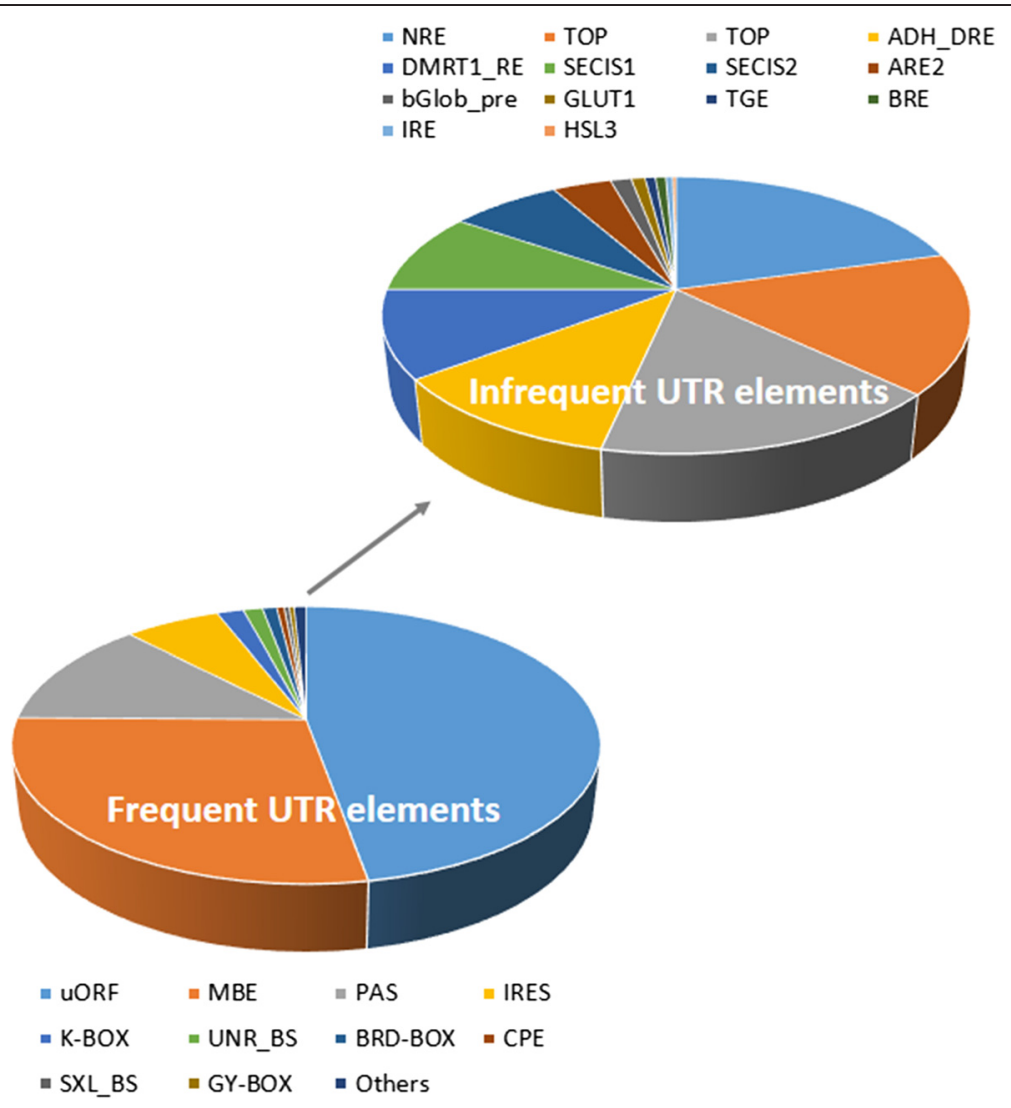

Fig. 5 Known regulatory elements in UTR-linked FPs. Lower pie chart: UORF, MBE, PAS, and IRES motifs are the most frequent regulatory sequences observed in UTR-linked FP sequences. Upper pie chart: Infrequently observed UTR elements contained in the "Others" category of the lower pie chart are shown 


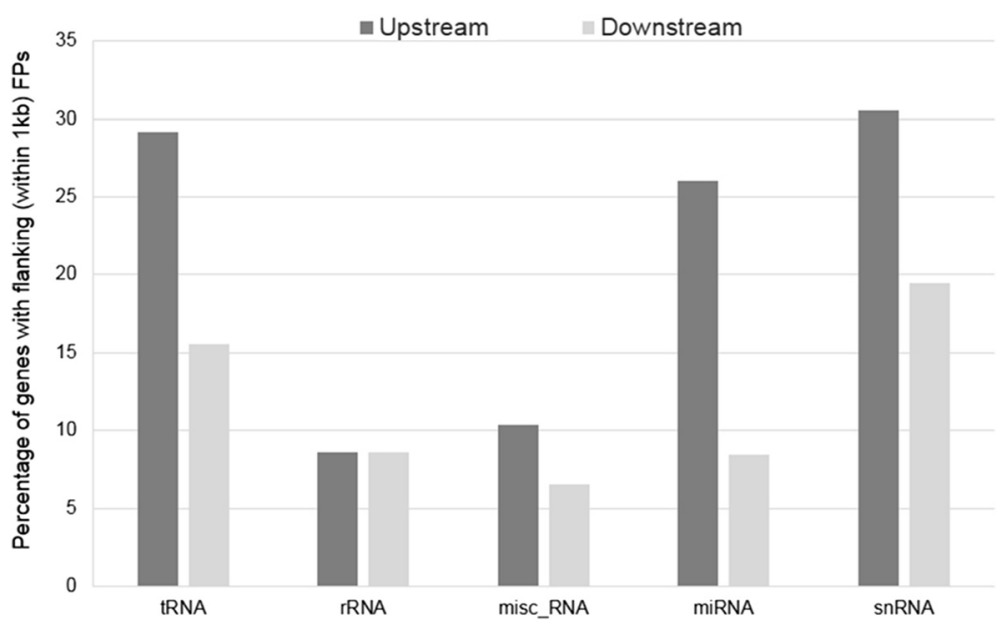

Fig. 6 FPs upstream of non-coding A. aegypti genes. tRNA, rRNA, snRNA, and microRNAs have FPs within 1 kb upstream of their TSSs

of $43 \mathrm{~A}$. aegypti miRNA genes may play a significant role in regulating synthesis of their precursors. Similarly, specific sequences upstream of tRNA genes are also known to regulate synthesis of tRNA molecules [48]. 287 FPs are associated with upstream sequences within $1 \mathrm{~kb}$ of $\sim 30 \%$ of A. aegypti tRNA genes, indicating possible cis-regulation of isoacceptor tRNAs. In addition to miRNA and tRNA, snRNA and rRNA genes have also been found to have association with cis-acting regulators [49, 50], and FPs were found to flank these genes in A. aegypti.

\section{Genetic variation in the regulatory regions of DENV susceptible and refractory mosquito strains}

Identifying and understanding genetic variation in noncoding regions, which is often challenging even in genetic model organisms, is particularly difficult in non-models. In a recent study, Illumina sequencing of $A$. aegypti Moyo-S and Moyo-R genomic DNA revealed genomewide genetic variation between the two strains [14-16]. In total, 13,158 high quality SNPs distributed throughout the genome (1321 supercontigs) were identified. These data were used to map the SNPs to FP sequences identified in the present study. The mapping data revealed that 3365 SNPs reside in FP DNA sequences (Additional file 11). To determine if there was any assortment bias of FAIREassociated SNPs, bootstrap randomized sampling was performed as described earlier [51] in which 10,000 FPs were randomly sampled to determine how many contained a SNP. Performing a 100,000 randomized sampling, it was determined that on average, 28 SNPs were detectable in every 1000 FPs (Additional file 12). This pattern was consistent in each repeat of randomization, clearly suggesting no bias in the assortment of SNPs in the FPs.

Although several intensive studies have assessed SNPs in $A$. aegypti in a genome-wide manner [52-54], our studies are to our knowledge the first attempt to map $A$. aegypti SNPs to regulatory sequences across the genome. Likewise, this investigation is to our knowledge the first to map genetic polymorphism data from pathogen susceptible and refractory host strains to non-coding regulatory regions across the genome of the host. FAIREseq could be used for comparable analyses in malaria vector mosquitoes or hosts for other disease-causing pathogens. As discussed by Meyer and Thye [55], the next steps will be to identify causative genetic variation and the functionality of associated factors. To this end, SNPs in putative $A$. aegypti regulatory sequences [14-16] that flank genes with differential transcriptome profiles in Moyo-S vs. Moyo-R strains are presently being functionally characterized.

\section{Conclusions}

The results of this investigation indicate that FAIREseq is a powerful tool for identification of regulatory DNA in the genomes of non-model or emerging model organisms, including human disease vector mosquitoes. In this study, FAIRE-seq analysis of open chromatin in $A$. aegypti embryos permitted genome-wide discovery of $>121,000$ regulatory elements throughout the genome of $A$. aegypti. Many of these sequences clustered in the $1 \mathrm{~kb} 5$ upstream flanking regions of genes known to be expressed at this stage. Known transcription factor consensus binding sites were enriched in the FPs, and all of the elements tested in vivo were confirmed to drive reporter gene expression in assays conducted in D. melanogaster transgenic animals. Of the $>13,000$ single nucleotide polymorphisms (SNPs) recently identified in DENV-susceptible and refractory mosquito strains, over one-quarter mapped to FPs, suggesting that genetic variation in regulatory sequences may contribute to the susceptibility/refractoriness of $A$. aegypti strains to DENV infection. 


\section{Supporting data}

Data sets supporting the results of this article are available within the article and its additional files, in the Sequence Read Archive (SRA) repository [56] (accession numbers SRP063665: http://www.ncbi.nlm.nih.gov/sra/SRP063665\% 5Baccn\%5D, SRX1046562: http://www.ncbi.nlm.nih.gov/ sra/SRX1046562\%5Baccn\%5D, and SRX1046561: http:// www.ncbi.nlm.nih.gov/sra/SRX1046561\%5Baccn\%5D), and in the VB Genome Browser. FPs can be visualized with the VB Genome Browser at the following link:http://www.vec torbase.org/Aedes_aegypti/Location/View?db=core; $r=$ super cont1.114:1965092-2050094; contigviewbottom=PRJNA294 762_FAIREseq_Scheel_AaegL3=tiling. Access to the $A$. aegypti scaffolds reference v.4 to which these FPs were mapped is available at: https://www.vectorbase.org/downloadinfo/aedes-aegypti-liverpoolscaffoldsaaegl3fagz.

\section{Additional files}

Additional file 1: Concordance of FAIRE-seq replicate experiments. IDR analyses demonstrated the concordance of FAIRE-seq replicate experiments. Correspondence curves of matched peaks describing the function between the number of peaks in common and the number of significant peaks between replicates (A), within replicates (C), and within the merge of all three replicates $(\mathrm{E})$ are shown. Correspondence curves of matched peaks describing the function between slope, representing the derivative, and illustrating the number of significant peaks between replicates (B), within replicates $(D)$, and within the merge of all three replicates (F) are shown. (PDF $146 \mathrm{~kb}$ )

Additional file 2: FP DNA Sequences. The A. aegypti supercontig locations (start-end sites) for the entire FAIRE-seq data set are provided. (XLSX $3291 \mathrm{~kb}$ )

Additional file 3: FPs immediately upstream of genes. FPs within $1 \mathrm{~kb}$ upstream of the TSS of the indicated genes are noted. FP numbers correspond to the numbers assigned in Additional file 2. (XLSX $185 \mathrm{~kb}$ )

Additional file 4: FPs mapping to $\mathbf{5}^{\prime}$ UTRs. FPs mapping to the $5^{\prime}$ UTRs of the indicated genes are noted. FP numbers correspond to the numbers assigned in Additional file 2. (XLSX $25 \mathrm{~kb}$ )

Additional file 5: FPs mapping to $3^{\prime}$ UTRs. FPs mapping to the $3^{\prime}$ UTRs of the indicated genes are noted. FP numbers correspond to the numbers assigned in Additional file 2. (XLSX $40 \mathrm{~kb}$ )

Additional file 6: Intragenic FPs. The number of intragenic FPs located within various genes is noted. FP numbers correspond to the numbers assigned in Additional file 2. (XLSX $243 \mathrm{~kb}$ )

Additional file 7: FPs mapping to exons. Genes with exonic FPs and the FP positions within exons are noted. FP numbers correspond to the numbers assigned in Additional file 2. (XLSX $14 \mathrm{~kb}$ )

Additional file 8: FPs mapping to introns. Genes with intronic FPs and FP positions within introns are noted. FP numbers correspond to the numbers assigned in Additional file 2. (XLSX $44 \mathrm{~kb}$ )

Additional file 9: FPs mapping to intron-exon boundaries. The intron-exon boundary locations of FPs in the indicated genes are noted. FP numbers correspond to the numbers assigned in Additional file 2. (XLSX $174 \mathrm{~kb})$

Additional file 10: FPs associated with non-coding genes. FPs within $1 \mathrm{~kb}$ upstream of the indicated non-coding genes are noted. The distance of the FP from the TSS of each gene is indicated. FP numbers correspond to the numbers assigned in Additional file 2. (XLSX $163 \mathrm{~kb}$ )

Additional file 11: Moyo-S vs. Moyo-R SNPs mapping to FPs. Moyo-S vs. Moyo-R SNPs at the indicated positions mapped to the FPs. The supercontig location of each SNP and corresponding FP ID numbers are indicated. FP numbers correspond to the numbers assigned in Additional file 2. (XLSX $181 \mathrm{~kb}$ )

Additional file 12: Random sampling of FPs. Bootstrap randomized sampling indicated that on average 25 SNPs were detectable in every 1000 FPs. (PDF 199 kb)

\section{Abbreviations}

CRE: cis-regulatory element ; CLOVER: cis-eLement OVER representation; DENV: dengue virus; EGFP: enhanced green fluorescent protein; FAIREseq: formaldehyde-assisted isolation of regulatory elements paired with DNA sequencing; FP: FAIRE peak; IDR: irreproducible discovery rate; IRES: internal ribosome entry site; LVP-IB12: Liverpool-IB12; MBE: Musashi-binding element; PAS: polyadenylation signal; SNP: single nucleotide polymorphism; TF: transcription factor; TSS: transcription start site; UORF: upstream open reading frame; UTR: untranslated region; VB: vectorbase.

\section{Competing interests}

The authors declare that they have no competing interests.

\section{Authors' contributions}

JS performed pre-processing and bioinformatics analysis of the FAIRE sequences and helped draft the manuscript. SB performed downstream analyses of FAIRE sequences and drafted the manuscript. PL performed FAIRE experiments and generated the constructs for transgenic reporter generation. KM assisted with characterization of reporters. DWS participated in sequence analyses and edited the manuscript. SE oversaw the bioinformatics analyses, participated in the design and coordination of the experiments, and helped draft the manuscript. MDS conceived of the study and led its design and coordination; she assisted with FAIRE DNA preparation, generation and analysis of transgenic reporters, as well as drafting and editing of the manuscript. All authors read and approved the final manuscript.

\section{Acknowledgements}

We are grateful to the staff of VectorBase and the University of Notre Dame Genomics and Bioinformatics Core Facility for their assistance. Thanks to Emily Andrews for assistance with the egg collections. Drosophila transgenics were generated by Rainbow Transgenic Flies, Inc. (Camarillo, CA). Open chromatin profiling was performed with a pilot grant to MDS from the Indiana Clinical and Translational Sciences Institute which is funded in part by Grant Number UL1TR001108 from the National Institutes of Health, National Center for Advancing Translational Sciences, Clinical and Translational Sciences Award. A Navari Family Foundation grant to MDS supported analysis of FP sequences. An NIH-NIAID R21Al117145-01 to MDS supported reporter generation and analysis. The University of Notre Dame Genomics and Bioinformatics Core Facility provided further support for this project. The funding bodies did not play roles in the experimental design, collection, analysis, or interpretation of data, writing of the manuscript or decision to submit it for publication.

\section{Author details}

${ }^{1}$ Eck Institute for Global Health, University of Notre Dame, Notre Dame, IN 46556, USA. ${ }^{2}$ Department of Biological Sciences, University of Notre Dame, Notre Dame, IN 46556, USA. '3 Department of Medical and Molecular Genetics, Indiana University School of Medicine, 1234 Notre Dame Ave., South Bend, IN 46617, USA. ${ }^{4}$ Department of Computer Science and Engineering, University of Notre Dame, Notre Dame, IN 46556, USA.

Received: 16 December 2015 Accepted: 12 February 2016 Published online: 10 May 2016

\section{References}

1. CDC: division of vector-borne diseases (DVBD), http://www.cdc.gov/ncezid/dvbd/. Accessed 15 June 2015

2. Holt RA, Subramanian GM, Halpern A, Sutton GG, Charlab R, Nusskern DR, et al. The genome sequence of the malaria mosquito Anopheles gambiae. Science. 2002;298:129-49.

3. Nene V, Wortman JR, Lawson D, Haas B, Kodira C, Tu ZJ, et al. Genome sequence of Aedes aegypti, a major arbovirus vector. Science. 2007;316: $1718-23$. 
4. Arensburger P, Megy K, Waterhouse RM, Abrudan J, Amedeo P, Antelo B, et al. Sequencing of Culex quinquefasciatus establishes a platform for mosquito comparative genomics. Science. 2010;330:86-8.

5. Neafsey DE, Waterhouse RM, Abai MR, Aganezov SS, Alekseyev MA, Allen JE, et al. Mosquito genomics. Highly evolvable malaria vectors: the genomes of 16 Anopheles mosquitoes. Science. 2015;347:1258522.

6. Sieglaff DH, Dunn WA, Xie XS, Megy K, Marinotti O, James AA. Comparative genomics allows the discovery of cis-regulatory elements in mosquitoes. Proc Natl Acad Sci U S A. 2009;106:3053-8.

7. Simon JM, Giresi PG, Davis IJ, Lieb JD. Using formaldehyde-assisted isolation of regulatory elements (FAIRE) to isolate active regulatory DNA. Nat Protoc. 2012;7:256-67.

8. Giresi PG, Kim J, McDaniell RM, lyer VR, Lieb JD. FAIRE (Formaldehyde-Assisted Isolation of Regulatory Elements) isolates active regulatory elements from human chromatin. Genome Res. 2007:17:877-85.

9. Giresi PG, Lieb JD. Isolation of active regulatory elements from eukaryotic chromatin using FAIRE (Formaldehyde Assisted Isolation of Regulatory Elements). Methods. 2009:48:233-9.

10. Henikoff S. Nucleosome destabilization in the epigenetic regulation of gene expression. Nat Rev Genet. 2008;9:15-26.

11. Thathy V, Severson DW, Christensen BM. Reinterpretation of the genetics of susceptibility of Aedes aegypti to Plasmodium gallinaceum. J Parasitol. 1994; 80:705-12.

12. Schneider JR, Mori A, Romero-Severson J, Chadee DD, Severson DW. Investigations of dengue-2 susceptibility and body size among Aedes aegypti populations. Med Vet Entomol. 2007;21:370-6.

13. Behura SK, Gomez-Machorro C, Harker BW, de Bruyn B, Lovin DD, Hemme $\mathrm{RR}$, et al. Global cross-talk of genes of the mosquito Aedes aegypti in response to dengue virus infection. PLoS Negl Trop Dis. 2011;5:e1385.

14. NCBI Aedes aegypti variation. http://www.ncbi.n/m.nih.gov/bioproject/ PRJNA269147. Accessed 3 Dec. 2014.

15. NCBI Moyo-S sequences. http://www.ncbi.nlm.nih.gov/sra/ SRX1046562\%5Baccn\%5D. Accessed 23 June 2015.

16. NCBI Moyo-R sequences. http://www.ncbi.nlm.nih.gov/sra/ SRX1046561\%5Baccn\%5D.Accessed 23 June 2015.

17. McKay DJ, Lieb JD. A common set of DNA regulatory elements shapes Drosophila appendages. Dev Cell. 2013;27:306-18.

18. Bolger AM, Lohse M, Usadel B. Trimmomatic: a flexible trimmer for Illumina sequence data. Bioinformatics. 2014;30:2114-20.

19. Babraham bioinformatics b FastQC. http://www.bioinformatics.bbsrc.ac.uk/ projects/fastqc/. Accessed 1 Oct. 2014.

20. Megy K, Emrich SJ, Lawson D, Campbell D, Dialynas E, Hughes DST, et al. VectorBase: improvements to a bioinformatics resource for invertebrate vector genomics. Nucleic Acids Res. 2012;40:D729-34.

21. Vectorbase: Vectorbase bioinformatics resource for invertebrate vectors of human pathogens. https://www.vectorbase.org/. Accessed 15 Jan. 2012.

22. Li H, Durbin R. Fast and accurate short read alignment with BurrowsWheeler transform. Bioinformatics. 2009:25:1754-60.

23. Choudhury O. Cooperative-computing-lab/cctools/makeflow_bwa_wrapper py. https://github.com/cooperative-computing-lab/cctools/blob/master/ galaxy/makeflow_bwa_wrapper.py. Accessed 1 Oct. 2014

24. Li QH, Brown JB, Huang HY, Bickel PJ. Measuring reproducibility of high-throughput experiments. Ann Appl Stat. 2011;5:1752-79.

25. Kundaje A. ENCODE: TF ChIP-seq peak calling using the irreproducibility discovery rate (IDR) framework. https://sites.google.com/site/anshulkundaje/ projects/idr. Accessed 1 Oct. 2014.

26. Zhang Y, Liu T, Meyer CA, Eeckhoute J, Johnson DS, Bernstein BE, et al. Model-based analysis of ChIP-Seq (MACS). Genome Biol. 2008;9.

27. Frith $M C, F u Y T, Y u L Q$, Chen JF, Hansen U, Weng ZP. Detection of functional DNA motifs via statistical over-representation. Nucleic Acids Res. 2004;32:1372-81.

28. Behura SK, Severson DW. Bidirectional promoters of insects: genome-wide comparison, evolutionary implication and influence on gene expression. J Molecular Biol. 2015:427:521-36.

29. Akbari OS, Antoshechkin I, Amrhein H, Williams B, Diloreto R, Sandler J, et al. The developmental transcriptome of the mosquito Aedes aegypti, an invasive species and major arbovirus vector. G3-Genes Genom Genet. 2013;3:1493-509.

30. Grillo G, Turi A, Licciulli F, Mignone F, Liuni S, Banfi S, et al. UTRdb and UTRsite (RELEASE 2010): a collection of sequences and regulatory motifs of the untranslated regions of eukaryotic mRNAs. Nucleic Acids Res. 2010;38:D75-80.
31. Pesole G, Liuni S. Internet resources for the functional analysis of $5^{\prime}$ and $3^{\prime}$ untranslated regions of eukaryotic mRNA. Trends Genet. 1999;15:378.

32. Patel NH. Imaging neuronal subsets and other cell-types in whole-mount Drosophila embryos and larvae using antibody probes. Methods Cell Biol. 1994:44:445-87.

33. Clemons A, Haugen M, Le C, Mori A, Tomchaney M, Severson DW, et al. siRNA-mediated gene targeting in Aedes aegypti embryos reveals that frazzled regulates vector mosquito CNS development. PLoS One. 2011;6, e16730.

34. Haugen M, Flannery E, Tomchaney M, Mori A, Behura SK, Severson DW, et al. Semaphorin-1a is required for Aedes aegypti embryonic nerve cord development. PLoS One. 2011;6, e21694.

35. Sarro J, Andrews E, Sun L, Behura SK, Tan JC, Zeng E, et al. Requirement for commissureless2 function during dipteran insect nerve cord development Dev Dyn. 2013;242:1466-77.

36. NCBI bioproject Aedes aegypti accession PRJNA294762. http://www.ncbi.nlm nih.gov/bioproject/PRJNA294762. Accessed 4 Sept. 2014.

37. Gaulton KJ, Nammo T, Pasquali L, Simon JM, Giresi PG, Fogarty M, et al. A map of open chromatin in human pancreatic islets. Nat Genet. 2010;42: 255-9.

38. Song L, Zhang Z, Grasfeder LL, Boyle AP, Giresi PG, Lee BK, et al. Open chromatin defined by DNasel and FAIRE identifies regulatory elements that shape cell-type identity. Genome Res. 2011:21:1757-67.

39. Hannenhalli S. Eukaryotic transcription factor binding sites-modeling and integrative search methods. Bioinformatics. 2008;24:1325-31.

40. Penalva LO, Sanchez L. RNA binding protein sex-lethal (Sxl) and control of Drosophila sex determination and dosage compensation. Microbiol Mol Biol Rev. 2003;67:343-59.

41. Wilkie GS, Dickson KS, Gray NK. Regulation of mRNA translation by 5'- and 3'-UTR-binding factors. Trends Biochem Sci. 2003;28:182-8.

42. Hayden CA, Bosco G. Comparative genomic analysis of novel conserved peptide upstream open reading frames in Drosophila melanogaster and other dipteran species. BMC Genomics. 2008;9:61.

43. Bulger M, Groudine M. Enhancers: the abundance and function of regulatory sequences beyond promoters. Dev Biol. 2010;339:250-7.

44. Kowalczyk MS, Hughes JR, Garrick D, Lynch MD, Sharpe JA, Sloane-Stanley JA, et al. Intragenic enhancers act as alternative promoters. Mol Cell. 2012;45:447-58,

45. Kvon EZ, Kazmar T, Stampfel G, Yanez-Cuna JO, Pagani M, Schernhuber K, et al. Genome-scale functional characterization of Drosophila developmental enhancers in vivo. Nature. 2014;512:91-5.

46. Borel C, Deutsch S, Letourneau A, Migliavacca E, Montgomery SB, Dimas AS, et al. Identification of cis- and trans-regulatory variation modulating microRNA expression levels in human fibroblasts. Genome Res. 2011;21:68-73.

47. Chavali PL, Funa K, Chavali S. Cis-regulation of microRNA expression by scaffold/matrix-attachment regions. Nucleic Acids Res. 2011:39:6908-18.

48. Zhang G, Lukoszek R, Mueller-Roeber B, Ignatova Z. Different sequence signatures in the upstream regions of plant and animal tRNA genes shape distinct modes of regulation. Nucleic Acids Res. 2011;39:3331-9.

49. Sharp SJ, Garcia AD. Transcription of the Drosophila melanogaster 5S RNA gene requires an upstream promoter and four intragenic sequence elements. Mol Cell Biol. 1988;8:1266-74.

50. Gunderson SI, Knuth MW, Burgess RR. The human U1 snRNA promoter correctly initiates transcription in vitro and is activated by pse1. Genes Dev. 1990;4:2048-60.

51. Behura SK, Singh BK, Severson DW. Antagonistic relationships between intron content and codon usage bias of genes in three mosquito species: functional and evolutionary implications. Evol Appl. 2013;6:1079-89.

52. Bonizzoni M, Britton M, Marinotti O, Dunn WA, Fass J, James AA. Probing functional polymorphisms in the dengue vector, Aedes aegypti. BMC Genomics. 2013:14:739.

53. Juneja P, Osei-Poku J, Ho YS, Ariani CV, Palmer WJ, Pain A, et al. Assembly of the genome of the disease vector Aedes aegypti onto a genetic linkage map allows mapping of genes affecting disease transmission. PLoS Negl Trop Dis. 2014:8.

54. Evans BR, Gloria-Soria A, Hou L, McBride C, Bonizzoni M, Zhao HY, et al. A multipurpose, high-throughput single-nucleotide polymorphism chip for the dengue and yellow fever mosquito, Aedes aegypti. G3-Genes Genom Genet. 2015;5:711-8

55. Meyer CG, Thye T. Host genetic studies in adult pulmonary tuberculosis. Semin Immunol. 2014;26:445-53.

56. Kodama Y, Shumway M, Leinonen $\mathrm{R}, \mathrm{C}$ INSD. The sequence read archive: explosive growth of sequencing data. Nucleic Acids Res. 2012;40:D54-6. 